\title{
Constrictive Pericarditis Progressing to Cardiac Tamponade Due to Metastatic Squamous Cell Carcinoma of the Larynx-A Challenging Diagnosis
}

\author{
Aasem Abu Shtaya*, Shoshan Perek, Adi Kibari, and Shai Cohen \\ Department of Internal Medicine “B”, Lady Davis Carmel Medical Center, Haifa, Israel
}

*Corresponding author: Shtaya AA, Department of Internal Medicine “B”, Lady Davis Carmel Medical Center, Haifa, Israel, E-mail: aasemab@ hotmail.com

Received: 21 Jun, 2019 | Accepted: 25 Jul, 2019 | Published: 30 Jul, 2019

Citation: Shtaya AA, Perek S, Kibari A, Cohen S (2019) Constrictive Pericarditis Progressing to Cardiac Tamponade Due to Metastatic Squamous Cell Carcinoma of the Larynx- A Challenging Diagnosis. J Clin Case Stu 4(2): dx.doi.org/10.16966/2471-4925.189

Copyright: (C) 2019 Shtaya AA, et al. This is an open-access article distributed under the terms of the Creative Commons Attribution License, which permits unrestricted use, distribution, and reproduction in any medium, provided the original author and source are credited.

\begin{abstract}
Effusive-Constrictive pericarditis is a challenging diagnosis due to its probable acute presentation as cardiac tamponade leading to quick deterioration of cardiac filling and pumping capacity impeded by an external force. The clinical picture can simulate other causes of fluid overload and decreased cardiac output making the diagnosis more complex. Most cases are idiopathic or due to a supposed predisposing viral infection. One of the often forgotten rare causes of this disease is primary occult malignancy of the pericardium or a metastatic carcinoma of other origins. We present a case of effusive-constrictive pericarditis advancing to cardiac tamponade due to extensive infiltration of the pericardium by metastatic squamous cell carcinoma of the larynx.
\end{abstract}

The case highlights the importance of taking into consideration the patient's past history and the less probable causes of pericardial effusion when there is no improvement on conventional therapy and avoiding misguidance by other common etiologies.

Keywords: Constrictive pericarditis; Tamponade; Squamous Cell Carcinoma; Larynx

\section{Learning Points}

- Constrictive pericarditis mimics heart failure with preserved ejection fraction in many aspects, but it is distinct in terms of etiology, prognosis and treatment.

- Malignancy is considered to be a rare cause of pericardial effusion, yet a common cause of symptomatic and hemorrhagic pericardial effusions.

- Metastatic squamous-cell carcinoma is a rare but still a possible cause of pleural and pericardial effusions. Rare other causes of the patients' disease are often forgotten because of more obvious and common etiologies that could explain the picture.

\section{Case Description}

A 69-year-old man presented with dyspnea and localized right chest pain that worsened gradually over the course of 3 weeks prior to admission. The patient's medical history is significant for essential hypertension, type II diabetes, and dyslipidemia. Three months before the current admission, the patient presented with non-ST elevation myocardial infarction, percutaneous coronary angiography revealed triple vessel disease requiring coronary artery bypass graft surgery that was performed on-pump with an ordinary post-operative course. Three years prior he was diagnosed with squamous cell carcinoma of the right vocal cord infiltrating adjacent cartilage; he successfully underwent cordectomy with adjuvant chemoradiotherapy, later on he was evaluated once a year through direct laryngoscopy and was considered cancer-free after three negative laryngoscopies.

The patient indicated suffering from dyspnea and localized sharp chest pain that started over prior three weeks worsening with time; the symptoms worsened with physical exercise and were associated with a productive cough and general fatigue.

Examination revealed normal vital signs, increased JVP, decreased air entry to the right lung with minimal pitting pedal edema. ECG showed normal sinus rhythm, no tachycardia and no evidence of ischemic changes; Chest $\mathrm{X}$-ray revealed a large unilateral right pleural effusion (Figure 1). Thoracentesis was performed draining $3 \mathrm{~L}$ of serous fluid with exudative parameters according to the Lights criteria. The fluid was negative for bacteria, cytology revealed rare atypical cells, the ADA levels were low. At this point, treatment with high dose aspirin and colchicine were started with a working diagnosis of post-cardiac injury syndrome, also treatment with furosemide was initiated due to volume overload.

Two weeks later, the patient presented again with worsening of dyspnea, orthopnea, a dry cough, abdominal fullness, and significant leg edema. Examination revealed a more prominent JVP, decreased 


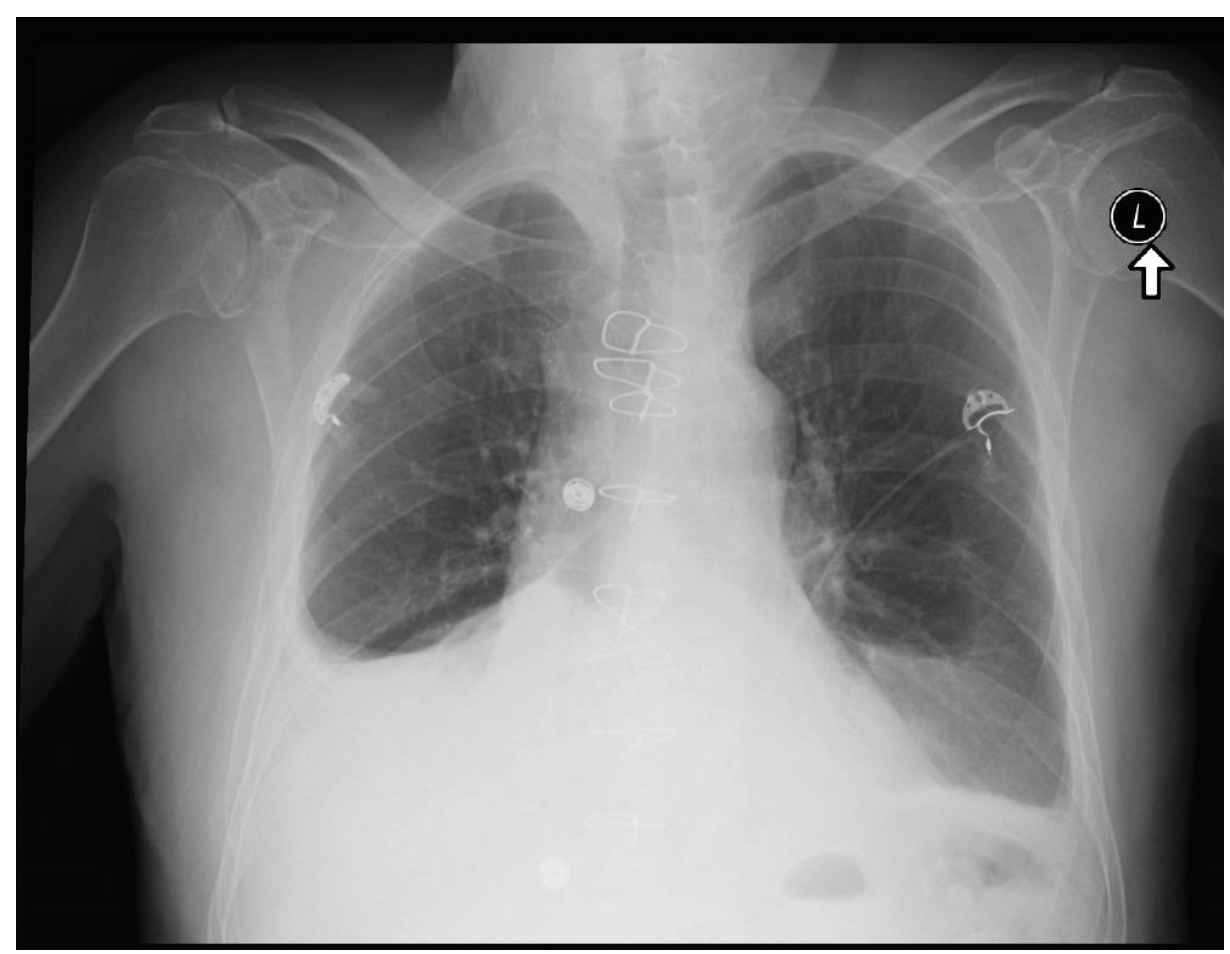

Figure 1: Chest X-ray showing mild to moderate right pleural effusion.

air entry to the right lung and $a+2$ bilateral pitting leg edema. Chest $\mathrm{X}$-ray revealed recurrent large right pleural effusion (Figure 2). Chest CT scan showed the same large right pleural effusion and additional small pericardial effusion, no pericardial thickening, no enlarged lymph nodes and no other additional finding. Recurrent thoracentesis was performed revealing the same prior findings without evidence of underlying etiology. A transthoracic echocardiogram revealed a minimal diastolic dysfunction, enlarged left atrium, minimal pericardial effusion, no respiratory flow variation and an enlarged inferior vena cava $(2 \mathrm{~cm})$ with non-significant collapse during inspiration indicating high right atrial pressure.

Assuming the patient had acute decompensated heart failure secondary to the aforementioned diastolic dysfunction treatment with high dose diuretics was initiated with minimal improvement, NT-Pro BNP levels were above 4,000. A follow-up chest X-ray was performed revealing bilateral pleural effusion, further thoracentesis through a Pigtail catheter revealed transudative effusion. Treatment with spironolactone and metolazone were added to the regimen. A consecutive transthoracic echocardiogram showed augmentation of the pericardial fluid, enlargement of both right and left atrium with an exacerbated enlargement of the inferior vena cava $(2.3 \mathrm{~cm})$ associated with non-significant inspiratory collapse. Due to those echocardiographic finding effusive constrictive pericarditis was suspected, the patient was transferred to the coronary care unit and was planned for a right heart catheterization.

The subsequent day the patient's condition deteriorated, with development of signs of hemodynamic shock. Examination revealed low blood pressure, desaturation on room air, tachycardia, a more pronounced JVP, muffled heart sounds and aggravation of peripheral edema.
Treatment with diuretics was held and instead IV fluids were initiated along with IV dopamine. An Echo-guided pericardiocentesis through a sub-xiphoid approach was performed draining $150 \mathrm{ml}$ of bloody discharge and revealing a thrombus within the pericardial space (Figure 3), without significant improvement in the hemodynamic status.

Because of hemodynamic instability the patient underwent emergent pericardiectomy. Pericardial tissue was extracted and sent for histopathology revealing fibro-fatty tissue extensively infiltrated by squamous cell carcinoma.

Postoperatively, the patient had a complicated course requiring continuous pharmacological ionotropic support; later on, he was connected to the extracorporeal membrane oxygenation device and underwent continuous renal replacement therapy due to volume overload.

Treatment with levosimendan was added as a weaning strategy from ECMO without success; the patient's general condition continued to deteriorate leading to multi-organ system failure, and eventually patient passed away ten days following the pericardiectomy.

\section{Discussion and Conclusion}

Constrictive pericarditis mimics heart failure with preserved ejection fraction in many aspects, but it is distinct in terms of etiology, prognosis and treatment. Scarring and consequent loss of elasticity of the pericardium usually results in impedance of cardiac filling leading to multiple hemodynamic sequelae. Diagnosing this disorder requires a high index of suspicion; while history and physical examination are of the paramount value for accurate diagnosis often accompanied by different modalities of cardiac imaging and even 


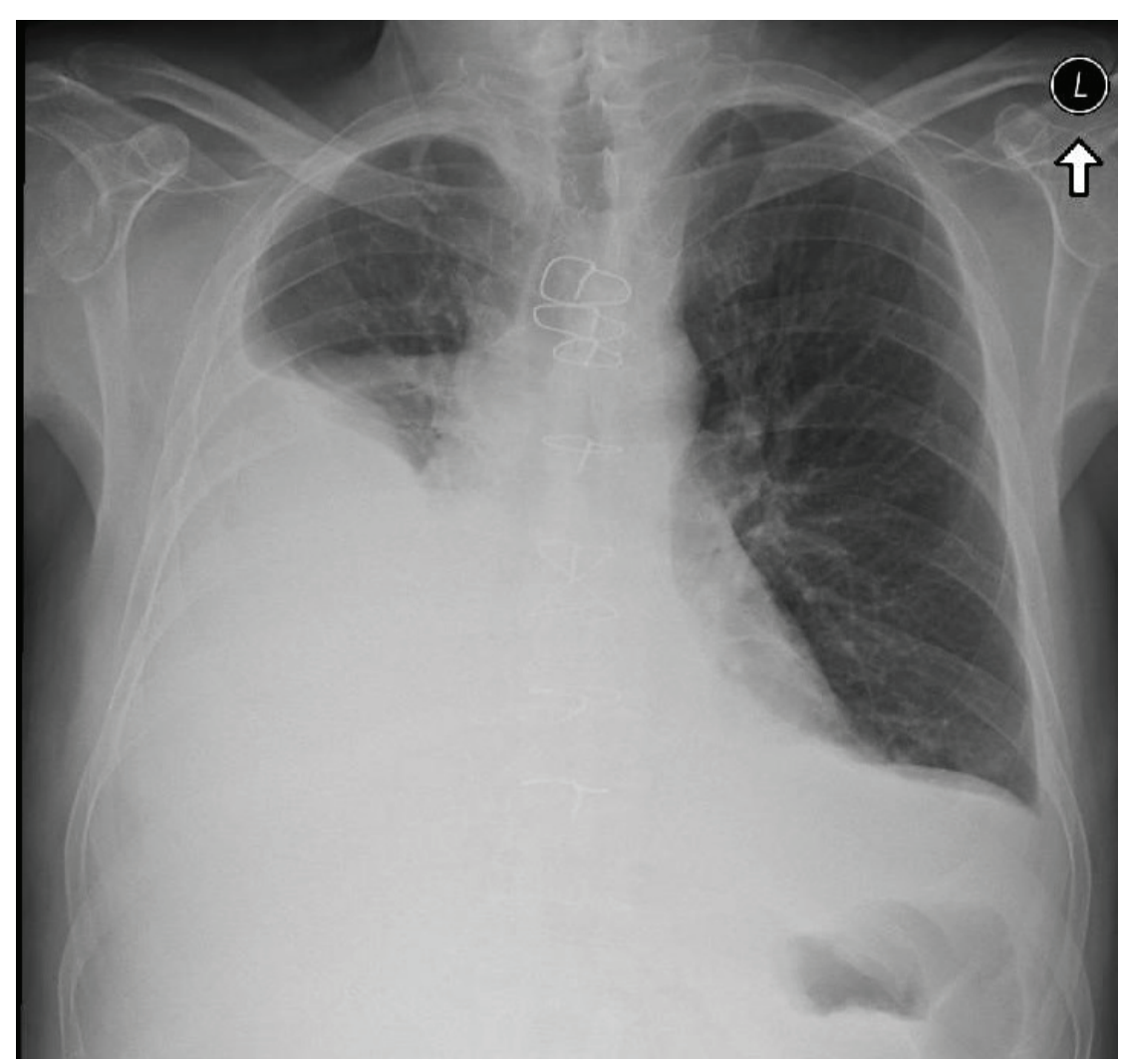

Figure 2: Chest X-ray showing augmentation of tight pleural effusion despite treatment.

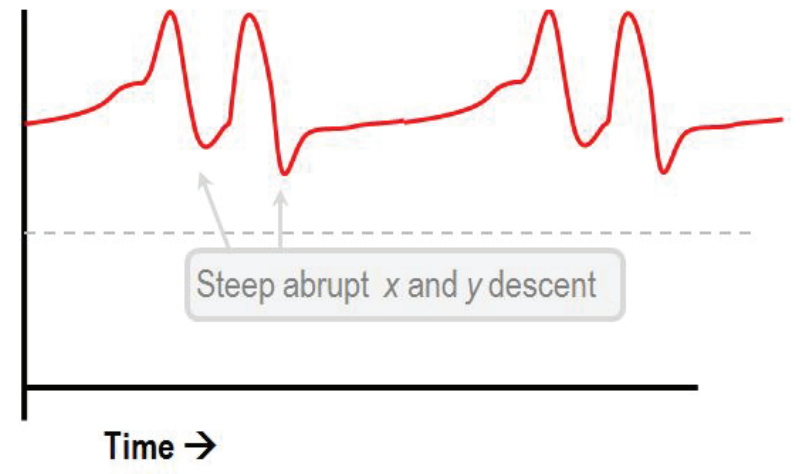

Figure 3: Jugular venous pressure waveform showing prominent $x$ and $\mathrm{y}$ descents of venous and atrial pressure.

invasive hemodynamic assessment. Recognition of the disorder and its underlying etiology is necessary for setting a treatment plan [1].

Tuberculosis was historically the most common cause of constrictive pericarditis in North America [2], but now considered to be a rare cause with reports ranging from less than $1 \%$ to $5.6 \%$ of cases [3]. The three dominant etiologies reported now in North America and Europe are idiopathic, prior cardiac surgery/pericardiotomy, and radiation therapy. The remaining cases are due to rheumatologic disease, infection, malignancy, trauma and other, rarer causes (Table 1).
Pericardial effusions due to malignancy are a severe manifestation of neoplasia of different origins; it is associated with significantly decreased patient survival [4]. Symptomatic patients can often progress to cardiac tamponade, which can rapidly lead to cardiovascular collapse or death. Thus, rapid diagnosis and timely treatment are necessary. Establishing the etiology of pericardial effusion remains challenging and difficult.

Malignancy is considered to be a rare cause of pericardial effusion, yet a common cause of symptomatic and hemorrhagic pericardial effusions (Table 2). Often, it can develop secondary to solid tumors (lungs and breast cancer, adenocarcinoma, squamous cell carcinoma, and melanoma) or hematological malignancies most often leukemia [5]. Flood SP, et al. described a rare case of signet cell adenocarcinoma of intestinal origin presenting as constrictive pericarditis due to infiltration of the pericardium [6] another case report published a few years ago demonstrated squamous cell carcinoma of the lungs presenting as a malignant pericardial effusion [7].

Primary neoplasms of the pericardium are much less common; they include malignant mesotheliomas, fibrosarcomas, lymphangiomas, hemangiomas, teratomas, neurofibromas, and lipomas. Malignant pericardial effusion may develop by several different pathophysiological mechanisms arising from either a direct extension or metastatic spread of the underlying malignancy via lymphocytes and blood or because of lymphatic drainage obstruction; they can also be a result of antineoplastic therapy.

Review of the literature reveals very few reports of squamous cell carcinoma of the oral cavity with evidence of metastasis to the pericardium [8]. Squamous cell carcinomas account for 90 to 95 
Table 1: Etiologies of constrictive pericarditis.

\begin{tabular}{|c|c|}
\hline Etiology & Reported Incidence \\
\hline Idiopathic or viral & 42 to 61 percent \\
\hline Post-cardiac surgery & 11 to 37 percent \\
\hline Post-radiation therapy & 2 to 31 percent \\
\hline Connective tissue disorder & 3 to 7 percent \\
\hline Postinfectious (tuberculous or purulent pericarditis) & 3 to 15 percent \\
\hline Miscellaneous causes (malignancy, trauma, drug-induced, asbestosis, sarcoidosis, uremic pericarditis) & 1 to 10 percent \\
\hline
\end{tabular}

Table 2: Etiologies of hemorrhagic pericardial effusion.

\begin{tabular}{|l|c|}
\hline \multicolumn{1}{|c|}{ Etiology } & \multicolumn{1}{|c|}{ Reported Incidence } \\
\hline Malignancy & 18 percent \\
\hline Percutaneous interventional procedures & 13 percent \\
\hline Post-pericardiotomy syndrome & 11 percent \\
\hline Complications of myocardial infarction (free wall rupture, thrombolysis) & 10 percent \\
\hline Idiopathic & 22 percent \\
\hline Other causes (including uremia, aortic dissection, trauma, etc) & \\
\hline
\end{tabular}

percent of the lesions in the oral cavity and larynx. The most common sites of metastasis are the lungs followed by the liver and bone [9].

Metastatic squamous-cell carcinoma is a rare cause of pleural effusions and is even less common in pericardial effusions. A review of all pericardial effusions examined in the Cytology Service at Montefiore Medical Center over a 15-year (1980-1994) period was undertaken $(\mathrm{N}=251)$. Four cases with metastatic squamous-cell carcinoma were identified among 39 malignant effusions. Two patients with metastatic squamous-cell carcinoma presented with cardiac tamponade, and the other two cases had progressive cardiac failure [10].

Patients developing constrictive-effusive pericarditis typically present with symptoms of dyspnea, and peripheral edema attributed to fluid overload. Diminished cardiac output results in fatigability, dyspnea on exertion and often chest discomfort. The history in a patient suspected to have the disease should focus on possible underlying etiologies.

On physical examination, the majority of patients display elevated jugular venous pressure; other less common findings include pulsus paradoxus, Kussmaul's sign, a pericardial knock, peripheral edema, ascites, and/or cachexia.

Plasma BNP is frequently obtained but is of limited clinical diagnostic value; there is no threshold established in the literature to rule in or out the diagnosis of constrictive pericarditis. BNP is released in response to wall stretch which is limited in constrictive pericarditis frequently leading to limited elevation.

Imaging modalities are usually used in the process of diagnosing this disorder, chest X-ray frequently demonstrates pericardial calcifications; a finding that highly consistent with constrictive pericarditis, however, the majority of patients will not demonstrate this finding thus its absence does not rule out the disease.
Transthoracic echocardiography is the cornerstone diagnostic test in a patient suspected to have constrictive pericarditis. The American College of Cardiology (ACC)/American Heart Association (AHA)/ American Society of Echocardiography (ASE) guidelines and the European Society of Cardiology (ESC) guidelines recommend the use of echocardiography for the evaluation of all patients with the suspected pericardial disease.

Still, echocardiography can be a non-informative and invasive hemodynamic evaluation as right heart catheterization is occasionally needed to confirm the diagnosis. The major findings are increased right atrial pressure with prominent $\mathrm{x}$ and $\mathrm{y}$ descents of venous and atrial pressure (Figure 3), square root sign is often seen in RV and LV diastolic pressure tracing reflecting rapid early diastolic filling of the ventricles, followed by lack of additional filling due to compression in mid and late diastole.

The management of symptomatic pericardial effusions has two major components. Acute management and relieving hemodynamic compromise in a patient with signs of tamponade, and chronic management aiming to prevent re-accumulation of fluid that can result in recurrence of symptoms and even tamponade.

For highly symptomatic patients, urgent fluid removal is usually performed to prevent hemodynamic shock, the effusion is typically drained by percutaneous pericardiocentesis guided by echocardiography. Patients suffering recurrent effusion accumulations are usually managed by creation of a permanent pericardial window allowing drainage to other body cavities.

In cancer patients, "classic" constrictive pericarditis due to malignant involvement is unusual. Most cases fall into the effusiveconstrictive category for which it is often the effusion and not the constriction that is causing symptoms and requires management. 
Nevertheless, some patients with constrictive pericarditis may require surgical removal of the pericardium, provided that the prognosis from the malignancy justifies surgery. Pericardiectomy is the only definitive treatment option for patients with chronic constrictive pericarditis. Medical therapy (i.e., diuretics) may be used as a temporizing measure and for patients who are not candidates for surgery.

All in all, our patient was managed according to the recommended guidelines undergoing all of the diagnostic modalities presented in the discussion as soon as the diagnosis of constrictive-effusive pericarditis was suspected. Though our patient was treated for weeks before for presumed post-pericardiotomy syndrome with no significant improvement; later he was treated for presumed fluid overload secondary to a diastolic dysfunction due to the initial echocardiographic findings showing mainly atrial enlargement along with relaxation dysfunction.

Looking for other possible causes of constrictive-effusive pericarditis or cardiac tamponade other than the apparent causes is of great importance, we believe an earlier pericardiocentesis with definitive cytological diagnosis would've changed the course of the patients' disease.

\section{References}

1. Welch TD, Oh JK (2017) Constrictive Pericarditis. Cardiol Clin 35 : 539-549.
2. Robertson R, Arnold CR (1962) Constrictive Pericarditis with Particular Reference to Etiology. Circulation 26: 525-529.

3. Ling LH, Oh JK, Schaff HV, Danielson GK, Mahoney DW, et al. (1999) Constrictive Pericarditis in the Modern Era. Circulation 100: 13801386.

4. Burazor I, Imazio M, Markel G, Adler Y (2013) Malignant Pericardial Effusion. Cardiology 124: 224-232.

5. Jeong TD, Jang S, Park CJ, Chi HS (2012) Prognostic Relevance of Pericardial Effusion in Patients with Malignant Diseases. Korean J Hematol 47: 237-238.

6. Flood SP, Ayah O, Furukawa S, Norris RB (2017) A Rare Cause of Constrictive Pericarditis. BMJ Case Rep 2017: 216813.

7. Nakazawa K, Kanemoto K, Suzuki H, Masuda M, Matsuno $Y$, et al. (2013) Purulent Pericarditis with Concurrent Detection of Streptococcus pneumoniae and Malignant Squamous Cells in Pericardial Fluid. Intern Med 52: 1413-1416.

8. Tsai YT, Kuo SW, Hao SP (2010) Cardiac Tamponade: A Rare Presentation from a Rare Metastatic Site in Oral Squamous Cell Carcinoma. Eur Arch Otorhinolaryngol 267: 1483-1485.

9. Garavello W, Ciardo A, Spreafico R, Gaini RM (2006) Risk Factors for Distant Metastases in Head and Neck Squamous Cell Carcinoma. Arch Otolaryngol Head Neck Surg 132: 762-766.

10. Hoda RS, Cangiarella J, Koss LG (1998) Metastatic Squamous Cell Carcinoma in Pericardial Effusion: Report of Four Cases, Two with Cardiac Tamponade. Diagn Cytopathol 18: 422-424. 